\title{
Neuropsychiatric perspectives on nodding syndrome in northern Uganda: a case series study and a review of the literature
}

\author{
Musisi $\mathrm{S}^{1 *}$, Akena $\mathrm{D}^{1}$, Nakimuli-Mpungu $\mathrm{E}^{1}$, Abbo $\mathrm{C}^{1}$, Okello $\mathrm{J}^{2}$
}

1. Department of Psychiatry, Makerere University College of Health Science, Kampala, Uganda

2. Department of Psychiatry, Gulu University Medical School, Gulu, Uganda.

\begin{abstract}
Background: Nodding Syndrome (NS), previously called Nodding Disease, is a chronic and debilitating illness affecting thousands of children aged 3-18 years in post-conflict Northern Uganda and South Sudan. Characterised by malnutrition, stunted growth, mental retardation and seizures, some researchers have designated it as epilepsy. With reports appearing in Northern Uganda in1997, NS reached epidemic proportions around 2000-2003 when people were moved into Internally Displaced People's (IDP) camps. Investigations for infections (onchocerciasis) and toxins have been inconclusive as to cause, treatment or outcome. No study has addressed the possible relationship of NS to childhood war-trauma experiences. Objective: To explore a possible relationship of exposure to prolonged war-trauma and the emergence of epidemic NS in Northern Uganda.

Method: This study was a case-series descriptive psychiatric naturalistic field observations of NS cases from homesteads in Northern Uganda and psychiatric investigations and treatment of NS cases referred to Mulago National Referral and Teaching Hospital.

Results: Detailed Psychiatric clinical evaluations and field observations revealed that NS children had been exposed to severe war-related psychological and physical trauma as well as non-specific CNS insults including untreated CNS infections / infestations and malnutrition possibly causing seizures. Many children suffered post-traumatic stress disorder (PTSD) and depression.

Conclusion: NS could present as an association of childhood complex PTSD, (called Developmental Trauma Disorder), occurring in the chronically war-traumatised children of Northern Uganda, complicated by severe prolonged depression with its characteristic symptoms of psychomotor retardation, anxiety, anhedonia and anorexia. This, coupled with food shortages, resulted in malnutrition, wasting and stunted growth with severe avitaminoses. Many children had seizures. All this calls for multi-disciplinary treatment approaches.
\end{abstract}

Key words: Nodding Syndrome, Post-traumatic Stress disorder, Epilepsy, Depression African Health sciences 2013; 13(2): 205 - 218 http://dx.doi.org/10.4314/ahs.v13i2.3

\section{Introduction}

Nodding Syndrome (NS), previously referred to as Nodding Disease, is a chronic and debilitating illness affecting thousands of children and adolescents mostly aged 3-18 years in post-conflict Northern Uganda and South Sudan ${ }^{1}$. It has claimed some lives. The first reports of NS in Northern Uganda appeared around 1997, but it reached epidemic proportions around 2000-2003 as cases became more rampant when people were moved into Internally Displaced People's (IDP) camps $^{1,2}$. However, as early as the 1960s, there had been reports of isolated cases of what appears to be NS

*Corresponding author:
Prof Seggane Musisi
Department of Psychiatry
College of Health Science Makerere University
Kampala, Uganda
Email: segganemusisi@yahoo.ca

African Health Sciences Vol 13 Issue 2 June 2013 in other parts of Uganda e.g. the Nakalanga Syndrome ${ }^{1,2,3}$. Since 2009, investigations particularly looking for infective and toxic causes have been conducted, but with no definitive conclusions as to the cause, treatment or outcome of NS 2,3,4,5. Extensive investigations have been carried out linking NS to infestation by onchocerciasis volvulus but they have all been inconclusive as have been numerous attempts at treatment using Ivermectin, antibiotics, deworming agents or vitamin replacements notably Pyridoxine ${ }^{2,3,4}$. A central argument has been whether to designate NS as an epileptic condition given that many NS children have had fits and seizure like activity on EEGs $1,2,3,4,5$, . However treatment with anticonvulsants, namely phenytoin, phenobarbitone , carbamazepine and recently Sodium valproate have not been solely and entirely effective, and no randomised clinical trials have so far been carried out, thus calling for other treatment measures including supportive treatments, feeding and 
psychiatric assessments and treatments ${ }^{6,7}$. Clinical case reports suggested that NS could be a syndrome of various causes including psychological and physical trauma as well as non-specific CNS insults including untreated CNS infections/infestations from a variety of agents ${ }^{7}$.

In 2011, the media and politicians from the Uganda Parliament became involved culminating in a January 2012 high level visit to Pader, Lamwo and Kitgum districts by a team composed of Ministry of Health $(\mathrm{MoH})$ officials, politicians and concerned academic researchers including those from the Makerere University Department of Psychiatry some of whom spoke Acholi, the local language.

\section{Methods}

The psychiatric team investigated the knowledge, attitudes and practices of the peoples of Northern Uganda to NS in addition to the psychiatric experience in treating NS cases referred to Mulago Teaching hospital. This paper reports on the descriptive naturalistic observations of three cases of NS observed in homesteads situated in Northern Uganda and the investigations and treatment of three of 8 cases by the Department of Psychiatry at Makerere University College Of Health Sciences at Mulago Hospital.

Three community-reported cases of NS were chosen to highlight the possible role of psychotrauma in association to the development of Nodding Syndrome. These were children with NS who were shown to Ministry of Health $(\mathrm{MoH})$ officials from Kampala by healthcare providers during a visitation by the $\mathrm{MoH}$ team from the $\mathrm{MoH}$ headquarters who were on a familiarisation tour of areas affected by NS in Northern Uganda.

The three cases from Mulago Hospital were chosen out of the 8 original cases referred to Mulago Hospital from Northern Uganda for thorough investigation of NS. The psychiatric team noted that Nodding Syndrome (luc-luc or yengo wic as coined by the locals) affected all the 8 cases referred to Mulago hospital and that all the 8 had had extreme trauma histories, were severely depressed and 7 were severely malnourished and stunted. Seven of these 8 cases improved with psychiatric interventions which included antidepressants (Imipramine), Family and play therapy as well as supervised feedings and continued anticonvulsant therapy using carbamazepine. The case that did not improve had brain damage, mental retardation, epilepsy in addition to the malnutrition and stunting. Although all the 8 cases had extensive trauma histories only 3 representative cases of the 8 were chosen to illustrate the possible role of psychotrauma in association to the development of Nodding Syndrome.

In this operational research, we used the etic-emic theoretical approach as most appropriate to deciphering the neuropsychiatric aspects of NS whose findings are presented below. The emic perspective helped us describe the "intrinsic cultural understandings of disease phenomena" $", 9$ as it related to the Northern Uganda community/society. It gave us the cultural explanatory model of the disease which influenced their health seeking behaviour ${ }^{9}$ and this approach enabled us to gather the Knowledge, Attitudes and Practices of the peoples of Northern Uganda in regards to NS. On the other hand, the etic perspective related to the objective observations that gave scientific meaning to observed phenomena and this enabled us to describe NS in terms understandable to the wider scientific community ${ }^{8,9}$ , e.g. the possible role of psychotrauma in the genesis of the observed complex neuropsychological symptoms of post-traumatic stress disorder (PTSD), depression, malnutrition, anxiety, conversion symptoms, dissociation, psychomotor retardation, catatonia, stupor, psychotic symptoms (hallucinations \& delusions), apparent Mental Retardation and convulsions. For psychiatric research, and especially cross-cultural psychiatric research, dichotomising the emic and etic approaches often falls short of giving the true picture and one often has to use mixed methods techniques ${ }^{8,9}$. NS lent itself appropriately to this, and we studied NS "as a community emergency" given the public outcry and political heat it had generated. The study was carried out in the form of descriptive case-studies. For purposes of this paper, 6 representative reports of the case-series is hereby presented: 3 from community homesteads and 3 from the 8 referrals to Mulago Hospital. The parents/guardians consented to studying and reporting of their children's stories.

\section{Findings: the case series}

\section{Community case studies of ns children seen in} their homesteads

\section{Homestead 1}

This was headed by MA, a single mother whose husband was gunned down in 2003 by the Lord's Resistance Army, LRA, rebels in the family's presence. Three of her children were subsequently affected by NS. One had died and two of them were 
interviewed. The family lived in IDP camps. The mother, MA, reported that NS had become a serious problem, that NS children burnt themselves and some had drowned. She also said that some NS children showed behaviour problems of defiance/ disobedience and not listening to parents. Prayers and medications had not contained the disease and that traditional healers had to be consulted "to chase away the 'cen', but symptoms still persisted". 'Cen' was the local term used to mean ghosts of dead people tormenting the living (who don't necessarily have had anything to do with their death). MA also said that NS affected people more during the days in the IDP camp but she complained that government officials were always in a hurry and gave no real solutions.

We observed NA, her daughter whom she called Aneno-Can (Translated as: I have seen/had a lot of problems). She was a 14 year old stunted girl who suffered from the typical nodding: she could not eat well, played with the food or showed no interest in it and finally threw it away. She would take off with the bowl of food, mumble some uncoordinated words but not eat. She claimed no recall of events just before she is served food till later on. She would then isolate herself and sleep for hours on end. Any food would trigger her nodding. Over time, NA had lost a lot of weight and her stature was stunted.

MA's other child, AN, was an 11 year old boy but much bigger than his stunted 14 year old sister NA. He started convulsing in 2003, at age 3 after the father's death at the hands of the Lord's Resistance Army (LRA) rebels. He described blurring of vision followed by a feeling that the world was turning upside down. After that, he said he had no recollection of events. He is told that he convulses. He said the attacks were worse at night and often he would fail to sleep. He said at times he got blurring of vision but didn't fall down, instead he described getting off his bike and walking about aimlessly, surprisingly though, he didn't wander in the bush, but followed the village path. He also claimed to have no recollection of such events. He said he got such episodes about once every 3-6 months if he was on medications of carbamazepine $200 \mathrm{mg}$ b.d but would get frequent episodes if he was off this medication. He said the disease (NS) did not spread, but it "had failed to cure".

MA's third child was an 18year old girl who had been ill since 2003, again following the father's killing by LRA rebels. She delivered at 17 years and she used to get the 'convulsing' episodes. She kept to herself. One day in late 2011, she went to the well on her own and drowned. It's unclear how this took place and the family declined to discuss it (? suicide).

\section{Homestead 2}

This was a home of two parents and six children. The husband, MK said that NS problem was associated with IDP camp life and he believed the disease could be infectious. He said that some NS children reported "seeing soldiers with pangas attacking them". MK said that older children were also getting affected alleging that the mode of transmission could be saliva. His wife felt that psychotherapy (talking) did not work. She wanted medications to be found to address the NS problem. Their affected child, AM, was a 14 year old stunted, wasted and now mentally retarded boy. He was the third affected child among his 6 siblings. The other two siblings had died earlier due to "this disease". The father (MK) wasn't willing to talk much about their death by drowning at the same time (? suicide pact). AM's problems, just like his siblings who had passed on, started in 2003 after a rebel attack of their IDP camp where some of his friends were abducted/killed. He would nod at their memory or at the site of food, when he would 'convulse' thereafter. He continued to worsen, and 'convulsed' repeatedly. He ate rarely and stopped talking about 2 years previously and now had to be "spoon" fed. The parents had resorted to tying him home for fear he would walk away in the bush or to the well and die (? like the others). They tied him to a tree so they could go to work and they kept an eye on him in turns. AM used separate utensils, plates and cups from the rest of the family as they feared the disease to be infectious. He could have up to 6 "convulsions" at night. He would froth at the mouth, urinated and passed stool on himself. He occasionally ate without incidence. AM was naked at the time that the family was visited and he had just had a 'convulsion', had passed urine on himself, and was reportedly 'unconscious' for all the period of almost an hour that the team was there!!

\section{Homestead 3}

This home was headed by BO, a jolly lady who reported that her husband had abandoned her 2 years previously, and would only 'visit' occasionally. She suspected the husband to have abandoned her because of the stress related to the disease (NS) and 
the problems she was having with it. BO had two affected children of her own (aged 15 and 13) and three from her co-wife (aged 12, 14 and 13) who had suspiciously died. She reported that the children's NS symptoms started in the year 2002 within months of each other after her co-wife died suddenly. She was not willing to discuss the cause of death of her co-wife but there was suspected poison. The first child started off by stretching his neck and he claimed a "lump" in it. They thought it was an ENT related problem for which a procedure was performed to 'correct' the throat disease but it "did not get cured" (? globus). Of her (BO) children, two nod and 3 just wander away. She reported numerous wrangles with neighbours because when the children wandered away, they got into trouble due to misbehaviours. Her children frequently assaulted others and were reportedly 'rough' when playing. She reported that her children ate a lot. None was stunted. She said that the medications only worked when the 'moon was up'. The symptoms continued worsening and within a year the whole family was affected involving her two children and the three of her co-wife. All the children had been in the IDP camps, none had specifically witnessed murders or been abducted. However they all knew of people who had been killed or had been abducted and the family always lived with this fear. She said the occurrence of this disease had been rare in the last few years after closing the IDP camps.

\section{Case studies of ns children seen in at Mulago hospital}

Case I: NS with epileptic seizures, MR, PTSD and depression

RO was a 12 year old Acholi boy from Bulbo village, Pader District. He presented with a history of generalised tonic and clonic seizures since early childhood. He got fits 2-3 times in a day which locally were referred to as ' jake or two oderu'. Once he fell in fire. His mother, an epileptic too, was unable to look after him and had abandoned him in the IDP camp. His father was unknown. He had no family and no caretaker in the camp. He never went to school. He had been unable to talk since he was abandoned by his mother 9 years previously. People in the camp would give him food and he slept anywhere. He was always sad, weak and had no appetite. He was severely stunted in growth. He could not walk and could not attend to his Activities of Daily Living (ADL).
The Physical Examination and Investigations revealed a wasted, malnourished and stunted young boy with multiple scars and glossitis. His FBC was normal but his electrolytes were low. Onchocerca volvulus tests were negative. EEG showed a non-specific epileptic pattern and the brain CT-scan showed nonspecific brain atrophy. EMG was not done.

The Mental Status Examination showed a severely malnourished and growth-stunted wasted young boy with multiple scars and wounds on the face at various stages of healing. He was dull, depressed and uncommunicative. He could not walk and had to be carried from place to place. He was observed to have grand-mal seizures while on the ward. Psychometric testing concluded that he had a low IQ in the moderately severe Mental Retardation range. The psychiatric diagnosis was Chronic/ Repeated Post Traumatic Stress Disorder (PTSD) in childhood; Epilepsy? cause; Mental Retardation (MR) and Depression probably causing him to have severe anhedonia and anorexia leading to much undernutrition and growth retardation.

In the camp the attempted treatment had been Carbamazepine, Deworming and antibiotics but with no improvement. At Mulago hospital the treatment included Carbamazepine, $200 \mathrm{mg}$ bd, Imipramine $25 \mathrm{mg}$ bd, feeding and supportive treatment with a constant attendant. There was some reduction in the seizures but no improvement in mood or behavior. It was concluded that RO suffered PTSD, depression, epilepsy and MR and that he had had brain damage although the cause could not be ascertained. Onchocerca volvulus skin snip tests were negative.

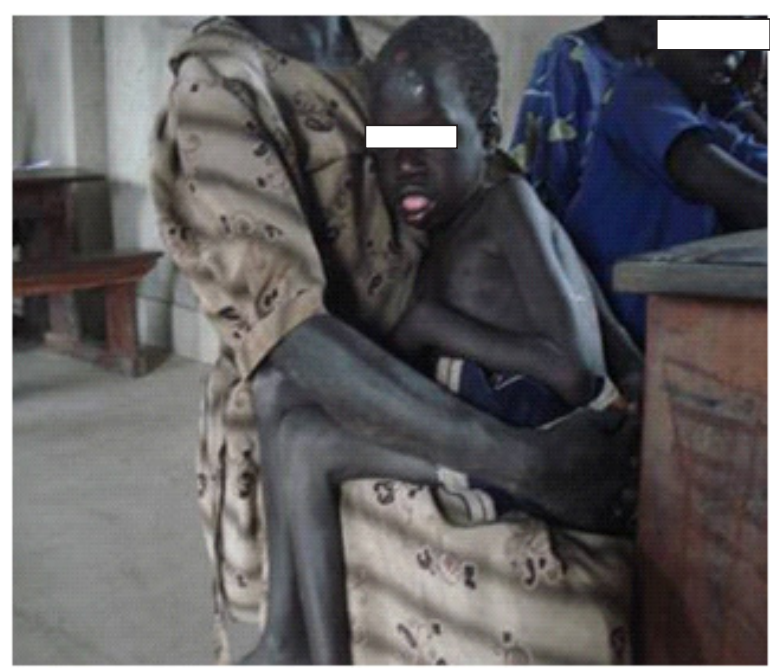


Case II: NS with complex PTSD and depression RW was a 14 year old boy an Acholi from Bulbo, Pader District. He presented with a 10 year history of tremors and spells of apparent loss of consciousness in which he would stare and be seemingly unresponsive to his surroundings and his head would then stoop which was referred to as nodding. At age of 4 years, he witnessed his father being shot dead and his brother abducted (and later killed) by rebel soldiers. He was the last born of six siblings and he lived with his peasant mother and the rest of siblings in the IDP camps. These camps were frequently attacked by rebels and he witnessed many people killed. At such times he would get "shocked, shook \& fainted with fear". This problem continued whenever he would get memories of these attacks or of his father's shooting and the abduction of his brother. He also always feared that he would also be abducted. He socially withdrew from people, stopped communicating, was always sad and isolated himself from others. He slept poorly with nightmares and always woke up feeling weak. He was always sickly, and progressively lost appetite and weight. He ate only once every two days and even then on strong persuasion and cajoling. He experienced significant wasting and growth retardation. He always had general weakness, tremors but no fits but had head stooping and stares called nodding by his mother and others. He could not walk and was always carried by his mother. He stopped school. He denied hearing voices or seeing any visions. He did not abuse drugs or alcohol.

On physical examination and investigations, there was nothing conclusive except for retarded growth. He weighed $30 \mathrm{Kg}$ at a height of $150 \mathrm{~cm}$ with a mid arm circumference of $16.8 \mathrm{~cm}$. His blood work, EEG and skin snips for Onchocerca Volvulus were all negative.

On mental status examination, he presented as a wasted young boy with stunted growth. He spoke very few words and was disinterested in his surroundings. Affectively, he was dull and depressed but not suicidal. There were no psychotic symptoms (no delusions and no hallucinations). He had poor motor coordination and could not walk alone unsupported. Cognitively he was slow with poor concentration and memory effort.

The Psychiatric diagnosis was Complex Post Traumatic Stress Disorder in Childhood with severe chronic depression leading to anhedonia and anorexia with subsequent undernutrition and growth retardation.
The treatment which was tried in the IDP camp included: Albendazol, Carbamazepine, Phenobarbitone, Antibiotics and Traditional healing but all with no improvement. At Mulago hospital, after psychiatric assessment, he was treated with Imipramine 50mg nightly, Family supportive therapy (counseling with mother) and encouraged play therapy as well as supervised feeding. After 4 weeks on the above treatment, his mood became brighter, he smiled, volunteered conversation, ate 2 full meals daily, slept well and the fear, stares and head stooping (nodding) all stopped. He was able to walk when supported though still tremulous on account of severe muscle wasting but he gained weight. He was then discharged as improved with advice to continue on the treatment.

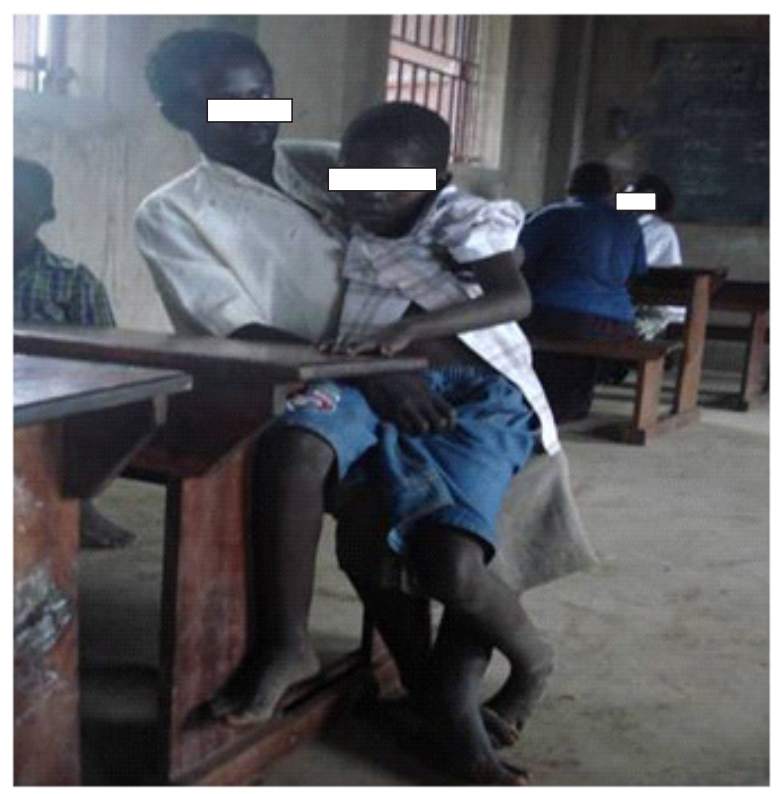

Final diagnosis: Complex PTSD in childhood with depression

\section{Case III: NS with depression and conversion disorder}

OR was a 14 year old Acholi from Awere, Pader district. He had lived in IDP camps with his parents and siblings since early childhood. He was doing well and attending school till both of his parents died 3 years previously of unknown causes. He was then looked after by his charismatic grandmother who believed that there was a spell cast on the family. Indeed, OR's older brother later just wandered away from home and was found dead in bush with his body being eaten by dogs. OR then developed "nodding and seizures" which occurred upto 4 times a day. Three of his other siblings also developed similar symptoms (familial clustering). OR felt sad, 
had poor sleep with bad dreams. His appetite was poor and he lost weight. His school performance deteriorated but he kept attending school anyway. He felt fed up with life but denied suicidal ideation. He also denied drug or alcohol abuse. He had had no psychiatric problems prior to these sad events to his family. He however described life in the IDP camps as particularly difficult. He was not ready to give details of these camp problems and dismissed any further symptoms on questionings.

On physical examination and investigations, he was found to be of normal physique for his age and sex and there was no stunting in his growth. All systemic examination was normal. Full Blood Count and electrolytes were within normal limits. EEG was normal. There was no Onchocercisis on skin snips. On Mental Status Examination, there were no grossly abnormal findings and it was essentially "normal". He was, however noted to be defensive, dismissive and he trivialized symptoms. He believed his grandmother's versions/assertions that a spell had been cast on his family, hence the unexplained deaths of his parents and brother. The Psychiatric diagnosis was depression with conversion symptoms on a background of multiple bereavements.

In the IDP camp the attempted treatment had included Carbamazepine, Deworming, Antibiotics and Traditional healers but with no improvement. At Mulago hospital the patient was treated with Imipramine $75 \mathrm{mg}$ nightly, Family and Individual supportive psychotherapy and brief grief therapy. Within two weeks, OR's mood brightened and he was able to talk readily about his grief problems as well as discuss the difficult life in the IDP camp. He asked for help for his other siblings whom he said had similar problems to him. His grandmother, however remained totally unconvinced about our treatments for the boy and insisted on returning to the Traditional healers "to the remove spell on the family". OR was discharged with advice to continue his treatment and to attend the Health Center in the IDP camp as well his siblings.

\section{Discussion and a review of the literature}

From our observations, investigations and treatments, we noted that nearly all NS children from Northern Uganda had had severe traumatic events in their lives. They had lived in IDP camps and had faced repeated traumas from which they could not escape thus creating a sense of severe chronic helplessness, fear and panic (anxiety). They had witnessed the brutal loss of loved ones or had their parents or siblings tortured, raped, abducted or killed. They had had severe social and medical deprivations, psychosocial under-stimulation and chronic food shortages. Many had developed severe Complex Post-Traumatic Stress Disorder (PTSD) in their childhood otherwise referred to as Developmental Trauma Disorder (DTD) and associated chronic depression with total anhedonia, helplessness, and with no hope. Our cases had severe loss of appetite (anorexia), a symptom of depression, as well as total inertia and psychomotor retardation to the point of a staring depressive stupor and/or catatonia. The loss of appetite and chronic food shortages led to severe malnutrition, avitaminoses and stunted growth. The poor health and sanitary conditions in the IDP camps meant that many could have suffered repeated untreated illnesses including CNS infections (malaria, meningitis, viruses etc) and other CNS insults e.g. from birth-trauma and/ or other brain traumas all of which could cause epileptic seizures. Many did not go to school or dropped out for a number of reasons: social, psychological and physical illness. There were no mental health interventions in the camps. The people resorted to unguided Traditional and Faith healing.

\section{Depression in Northern Uganda and NS}

High rates of depression amongst both war-affected adults and children have been reported in Northern Uganda $15,16,17,18,19$. The development of severe depression in traumatized children is well documented in the literature. Bowlby first described it as anaclactic depression ${ }^{20,21}$. It is a condition in young children who have experienced severe traumata with separation from their primary attachment figures (mothers). The children then go through various stages of mourning and finally severe depression with failure to thrive $\mathrm{e}^{20,21}$. These stages include: i) anxious behaviour ii) protest with intense crying and struggling iii) despair characterised as total helplessness, hopelessness and monotonous crying iv) mourning manifesting as profound depression, social detachment and withdrawal, sometimes presenting as stuporus staring v) failure to eat with failure to thrive resulting in stunting and wasting vi) preoccupation with inanimate objects and own body parts including genitalia vii) fecal and urine smearing, head banging, rocking, (convulsions) viii) marasmus and or kwashiorkor with eventual death from severe malnutrition which may be complicated by infections due to lowered immunity. Such depression was artificially reproduced in the famous Harlow's monkeys experiments who were deprived of their attachment figures ${ }^{22}$.

African Health Sciences Vol 13 Issue 2 June 2013 
In adolescents and adults, severe prolonged untreated depression can present as Cotard Syndrome ${ }^{23}$. This is a profound chronic depressive syndrome with psychomotor retardation, pseudo-dementia, somatic delusional interpretation of bodily function, nihilistic views of the world, catatonia, mutism, anhedonia, anorexia and severe wasting and stupor. Patients can even develop bedsores from prolonged immobility. Severe depression in traumatized children can have these manifestations depending on the age of the child. High rates of depression have been reported in the war-traumatised children of Northern Uganda and suicide is common ${ }^{16,19}$. Could this be the reason for the unexplained deaths by drowning, burning or just disappearing in the bush only to be found dead?

\section{Malnutrition and Stunted growth}

Failure to thrive with severe marasmus and kwashiokor has been seen in depressed malnourished African children especially when separated from their attachment figures even without a history of war-trauma (Mwanamugimu Child Nutrition ward, Mulago Hospital). In war situations, including Northern Uganda, malnutrition, especially amongst children is very prevalent. Tumwine (2012) reported high rates of malnutrition in the NS children of the cultivator Mundri tribe in South Sudan but not in the milk drinking cattle keeper Dinka tribe ${ }^{13}$. The NS children of Northern Uganda were severely malnourished and showed Protein-Calorie Malnutrition (Marasmic-Kwashiokor) with severe various vitamin deficiencies. No study of their neuropsychiatric manifestations has been carried out except for possible pyridoxine dependent seizures (vitamin B-6) and hence treatment trials of NS with pyridoxine $e^{4}$. Various neuropsychiatric presentations due to chronic vitamin deficiencies are well described in the literature e.g. Nicotinic Acid deficiency ${ }^{24}$. The cornerstone of the treatment interventions for NS in Northern Uganda has been feeding and replacement of those vital nutrients in the affected children with much success ${ }^{6}$. However, some of these malnourished children may forever remain stunted as they had already passed the age of maximum bone growth ${ }^{3}$.

\section{Post Traumatic Stress Disorder and NS:}

Post-traumatic stress disorder (PTSD) is the most common psychiatric disorder of severely traumatized individuals as happens in war. Often it is complicated by depression. High rates of PTSD have been reported in Northern Uganda ${ }^{15,16,17,18,19}$.
It has been stated that the prevalence of PTSD and traumatic depression affects far more people in Africa than malaria and HIV/AIDS combined. The NS syndrome children we saw had severe PTSD for all the time they had lived in IDP camps. On an almost daily basis they faced repeated traumas from which they could not escape thus creating a sense of severe chronic helplessness, anxiety and depression. They had witnessed the brutal loss of loved ones or had their siblings tortured, raped or abducted. High rates of PTSD have been reported by various researchers of the effects of war in Northern Uganda associated with high rates of depression and suicides 15, 16, 17, 18, 19. Kinyanda et al (2001) reported a suicide rate of 16-20 per 100,000 of population compared to 8 per 100,000 in non-war affected communities ${ }^{25}$. Ovuga et al (2005) found similar high rates of suicide in the war-affected communities of Northern $\mathrm{Uganda}^{26}$. These high suicide rates included children. It is therefore worth investigating for possible suicide in the NS children with high rates of PTSD and depression who had died suspiciously of drowning, burning or just wandering away in the bush only to be found dead. No psychological autopsy studies have been done on any NS deaths.

J. Herman ( 1997) described the concept of Complex PTSD as a result of protracted and repeated traumatization of an individual which leads to a complex process of psychological reactions where the existence of previously held values and views of the world is doubted leading to changes in personality, beliefs and distrust of everyone ${ }^{27}$. Depression, substance abuse and suicide attempts are common complications of PTSD and especially Complex PTSD. In children, situations of Complex PTSD exist but the manifest symptoms will depend on the age of child, the nature of the trauma, the repetition of the trauma, gender of the child and support systems available to the child ${ }^{28}$. Van Der Kolk (2005) has described Complex PTSD in children as Developmental Trauma Disorder (DTD) as a necessary concept towards the rational diagnosis of chronically traumatized children ${ }^{28}$. He emphasized that for one to clinically understand the developmental impact of childhood trauma in children, it was necessary to integrate the child's biological, psychological, and cultural perspectives ${ }^{29}$. This concept of developmental trauma disorder very aptly describes the complex PTSD symptoms found in the NS children at their various ages. This observation underpins the need for an emic 
approach to understanding NS as possibly secondary to the chronic trauma that the children were subjected to in Northern Uganda.

\section{"Cen" as the Cutural Interpretation of NS}

Complex symptoms of PTSD are often influenced by cultural underpinnings as possible culture bound PTSD syndromes ${ }^{30}$. Boehnlein, (2001) in a study of PTSD in different cultures explained the cultural interpretations of common physiological processes in PTSD $^{31}$. He concluded that by listening simultaneously to the literal (spoken) language, knowing cultural metaphors and observing somatic (body) language, one is led to a more comprehensive understanding of human suffering in the care of the traumatized ${ }^{31}$. This observation formed the basis of the emic approach to our study of NS in the Northern Uganda homesteads. The people of Northern Uganda recognized the emergence of the NS epidemic in association to the war with the LRA, but more specifically to being moved into IDP camps where they met indescribable suffering and torture at the hands of the fighting forces. They lost loved ones whom they never buried. Children got abducted. Many were killed in the process or their fathers and brothers shot in their presence for resisting the abduction. Women and girls were raped. The IDP camps were often raided and set ablaze. Hygienic conditions were deplorable. Infections were ripe. Food was always in short supply. Alcohol abuse was at its highest. Health infrastructure had broken down. There was hardly any medical care and certainly no psychiatric care. The people described NS as cen, an Acholi word for 'dead peoples' spirits which come to haunt the living for a variety of reasons" including improper burial. They also saw new cases of NS dwindle after the LRA was kicked out of Northern Uganda and the IDP camps were disbanded. The people of Northern Uganda correctly associated NS with war-trauma, the difficult IDP camp life and the spirits of the dead haunting the living. They described their NS children as haunted, bewitched, cursed, and they witnessed them die by drowning, burning or just walking away in the bush never to return. They tied their children on trees to stop this. They called NS "luc luc or yengo wic" and recognised it as a new disease in their midst. They did not call it epilepsy "jake or two oderu", these being more familiar terms to mean epileptic fits which they had always seen from time immemorial. These are very telling emic observations. Today, such severe and repeated chronic traumatization of children is referred to as Toxic stress.

\section{Seizures, Epilepsy and NS}

Many NS children suffered seizures. For long epilepsy has been known to be the most common neuropsychiatric disorder in Northern Uganda and indeed in all rural communities in Africa ${ }^{32}$. Indeed positive EEG findings have been found in association with $\mathrm{NS}^{2,5}$. However, NS was not called epilepsy by the locals even when they saw actual fitting. The local Acholi word for epilepsy is "jake or two oderu" and that for NS is "luc luc oryengo wic". That children with NS may have epilepsy is a certainty and a common finding previously referred to by health workers as "Nodding Syndrome Plus" . Some researchers have referred to NS as akinetic seizures $^{2,5}$. However, considering all children with the label of NS as having akinetic seizures does not explain all the observed phenomena in NS, hence the continued quest for causes of and treatment for NS. Scientifically linking NS to oncocerciasis volvulus has proved not possible. NS tends to start at age of 3 years. According to Piaget's developmental stages, this is the age when children have developed the cognitive ability of "object constancy"........to be able to keep permanent concepts of significant others (parents/caretakers) in their mindsets ${ }^{33}$. The traumatic loss of such will certainly lead to PTSD and depression or DTD depending on the child's age. That many NS children developed depression and NS after witnessing horrendous trauma is a common finding. These are important etic observations.

\section{NS in Eastern Africa}

Early reports by L. Jilek-Aall (1962) described children with attacks of "nodding head," with or withought "grand- mal" seizures in Ulanga district of Tanzania ${ }^{2,9}$. At that time, no reports associating NS with onchocerciasis were reported although it was noted that onchocerca volvulas was endemic in the Ulanga district of Tanzania ${ }^{9}$. More recent reports from Tanzania did not find CNS onchocerciasis in NS victims ${ }^{10}$. Recently Idro et al (2013) and Sejvar et al (2013) have described Clinical neurological, Electrophysiologic and Neuroimaging evidence linking NS to epilepsy ${ }^{2,5}$. These researchers concluded that NS was a variant of epilepsy. However, none of them described the role of psychotrauma in NS and they did not attempt to explain the psychiatric phenomena they found in their case-series. 
Closer at home, Kipp et al, (1994) in Eastern and Western Uganda reported on the Nakalanga syndrome consisting of epilepsy, stunted growth and mental retardation, reminiscent of the "nodding disease" 34 . The affected areas were in forest regions with fast flowing rivers where simulium black flies, the carriers of the onchcerciasis volvulus worm, thrived. Indeed spraying that eradicated these flies also eradicated onchocerciasis and the Nakalanga syndrome from these Mabira forest areas.

Reports from South Sudan reported familial clustering of cases of NS but failed to find significantly elevated onchocerciasis antibodies in the blood or CSF samples of the affected children ${ }^{11,12}$. Earlier investigations by J. Tumwine in South Sudan, had linked NS to onchocerciasis ${ }^{3,13}$. Tumwine had also noted that the disease was more rampant in communities where the Sudan Peoples' Liberation Army (SPLA) operations had taken place and in the Mundri cultivator tribe with much poverty but not in the cattle keeping Dinka tribe where children drank milk as a daily staple ${ }^{3}$. For the first time, this report linked the possible role of malnutrition to NS. Recent reports of traumatised children from many conflict ridden areas of the world including Asia, Africa and Latin America report much malnutrition, stunted growth and mental retardation as well as psychotraumatic complications in children affected by mass war-trauma ${ }^{14}$.

\section{NS in other areas}

It is important to note that epilepsy is a very common neuropsychiatric disorder in Africa and other developing countries. Also high rates of epilepsy have been reported in areas highly affected by onchocerca volvulus $^{5,10}$. In West Africa in 1983, Gerrits et al reported on children with "dropping the head in the pan" among the Bassa and Kpelle in Liberia ${ }^{1,10}$. Other areas have included South America (Columbia) , South Sudan, The Democratic Republic Of Congo and Ghana ${ }^{5}$. Again although these areas are endemic in onchocerciasis, a causative association of NS with onchocerciasis was not confirmed. Recentlty there have been suggestions of an autoimmune process triggered by the onchocerciasis worm, but discerning the mechanism has remained elusive ${ }^{3}$.

\section{NS surveillance in Uganda}

In Uganda, Onchocerciasis volvulus was reported to be endemic in the districts of Kyaggwe (Mabira Forest), Kabarole, Kibaale, Hoima, Acholi sub-region and $Z$ ombo ${ }^{2,5}$. These areas also have very high rates of epilepsy. Indeed the Nakalanga syndrome, mentioned earlier, was reported in Mukono district before the area was sprayed to clear it of the simulium black fly which carries the Onchocerca Volvulus worm. However reports of the epidemic NS in Northern Uganda only began to appear in around $1997^{1}$. In 2009, the Uganda Ministry of Health $(\mathrm{MoH})$ report summarised the problem of NS in Northern Uganda as follows:

"In the districts of Kitgum and Pader of Northern Uganda, a progressive disease characterized by head nodding, mental retardation and stunted growth was noticed for over five years before it was reported to Ministry of Health in 2009. Over 300 children in the districts had been affected by the strange condition. The disease escalated in 2003 when most of the Northern Uganda population had moved to Internally Displaced People's (IDP) Camps and predominantly affected children aged $5-15$ years, of both sexes but with a male preponderance ...."

NS became a major public health problem in the districts of Kitgum, Lamwo and Pader in Northern Uganda with the total number of cases there being 1,876 with the highest burden in Labongo Akwang sub-county in Kitgum district where the prevalence of NS was reported to be 1,305 per 100,000 population and in Pader district where the prevalence was 519 per 100,000 population ${ }^{1}$. There was a noted increase in the number of new cases over the years which peaked in 2008/9 at the height of the war against the LRA in Kitgum, Lamwo and Pader districts when people lived in IDP camps. 


\section{Box 1: Verbal reports given by parents/patients and village elders at the field visits}

\section{The Village Heath Team, VHT}

OJ a male VHT member had this to say about NS: "The diseases started way back in 1998. It was diagnosed as chronic but with little help at the government hospital. The current medications were not working. Between 2000 and 2003 when people were moved into the IDP camps, more children were affected and the symptoms became worse. In 2009 \& 2010 doctors did tests on the skin, blood and saliva but we not told the results. Also the distance to the Health Centres was too far".

A Female VHT member described three types of this disease : one with loss of consciousness and convulsions; the second type whereby children appeared dazed and sometimes fell down, the children would stare in space and sometimes wandered away like mentally disturbed people and some could convulse but very briefly, they didn't fall down. The third type was the nodding type and this manifested with stunting. This female VHT member said the children would actually grow normally initially but regressed later after onset of the disease and this was the real NS. She said the people of the affected village were not 'very clean' (lacked self care/hygiene) and she blamed this on the dirty water source at the river or the shallow well.

A TPO field worker felt that dispensing carbamazepine reduced the symptoms. He said the disease was wide spread to the entire Acholi region and that the children were actually very ill and they forgot quickly which impaired their learning. He noted that there was poor follow up of the affected children.

Two parents said that NS was an extensive problem triggered by food, and acknowledged the different types of diseases. The parents asked for help and said the medications helped reduce the symptoms but did not cure the disease. Their children still dosed, convulsed, and smeared themselves with food and passed stool and urine on themselves.

One parent, MO said that two of his children were affected by NS which he called 'luc-luc" in Acholi. He reported that his two children aged 13 and 15 developed the NS problems in 2003 when they went to the IDP camp. MO said his children were sent to Kampala at IHK and that he was shown 'atrophied' brain on their scans. He said the children did not improve in Kampala's IHK even after coming back from Kampala. He said the children had problems

\section{Box 2: Summary of psychiatric observations from the field visits}

The psychiatric team that accompanied the MoH observed that NS kids had the following:

1. Behavioural disturbances \& Suspicious deaths: "Wandering away from home, acting aggressively or violent; Some NS children died by drowning, self-burning or wandering in bush and were found dead. No cause of death could be established; some reported seeing people/soldiers attacking them with machetes and/or guns; they would scream and become very scared but no one else saw the attackers;

2. Psycho-trauma stories: All the NS syndrome children had severe PTSD for all the time they had lived in IDP camps. On an almost daily basis they faced repeated traumas from which they could not escape thus creating a sense of severe chronic helplessness, anxiety and depression. They had witnessed the brutal loss of loved ones or had their siblings tortured, raped or abducted. This was in keeping with the high rates of PTSD as has been reported by various researchers of the effects of war in Northern Uganda. There are also associated high rates of depression and suicides $^{15,16,17,18,19}$. It is therefore not surprising that the NS children with high rates of PTSD and depression died suspiciously of drowning, burning or just wandering away in the bush only to be found dead.

3. Depression: NS kids looked sad, depressed, withdrawn, wasted, apathetic and/or catatonic. Some would lose consciousness and convulse. Some had brain damage and mental retardation.

4. Malnutrition: Many NS children had severe malnutrition and avitaminoses with skin changes, wasted muscles, stunted growth and marasmic-kwashiokoir stigmata accompanied by neuropsychiatric complaints including slowed mentation, mental retardation and convulsions. Untreated, the condition incapacitated the child with some developing MR, convulsions, losing speech and sphincter control and having suspicious deaths.

5. Social Problems: Parents couldn't go to attend to their gardens for fear of their NS children wandering away from home \& dying in the bush by burning, drowning or just being found dead. So the parents resorted to tying the children onto trees. The community felt that NS was caused by "cen" which they explained as "dead people's spirits which came to haunt the living for a variety of reasons including improper burial”.

Conclusion: In Akwang camp and Mulago Hospital most children had improved by feeding and supportive care. The psychiatrists suggested a holistic approach in the treatment of NS involving psychiatrists, paediatricians, nutritionists, clinical psychologists, medical anthropologists, social workers and nurses all working together. 
In 2007-2009, a number of organizations including the Uganda Ministry of Health $(\mathrm{MoH})$ and the , Center for Disease Control (CDC), took keen interest and carried out a number of investigations on NS in Northern Uganda including toxicology $y^{2,3,4,5}$. Samples of saliva/urine/blood/skin snips were taken. Water sources were sampled as well as the soil; some insects were also captured and assessed as well as bush meat. The findings from these investigations were non-contributory, with no significant findings seen as to the aetiology of NS. Considered to be a progressive disorder of the nervous system with unknown aetiology, treatment and prognosis, NS began to be treated as an epileptic disorder but without total definitive success $s^{1,2,3,4,5}$. It was, nevertheless, concluded that the NS head nodding was atonic seizures in the category of symptomatic generalised epilepsy $y^{2,5,10}$.

NS has attracted much political attention since 2010 resulting in many sensationalised media stories $^{6}$ and alarmist concerns from all over the country. Consequently the Uganda Government set up NS treatment centers in Northern Uganda, such as the Atanga NS Treatment Center in Pader district ${ }^{6}$. Although the definitive cause(s) and treatment of NS has remained elusive to date, symptomatic and supportive treatment of NS cases has reported successes. Odongkara (2011), the Medical Director of the Atanga NS Treatment Center stated that ".... the Atanga NS Treatment Center in Pader district had registered 596 NS patients by May 2012 and of these 414 were diagnosed as NS after screening. With only supportive medical treatment and feeding, most of the victims recovered with no more head nodding and only 15 still remained symptomatic" . Odongkara went on to say that "Despite the various challenges, we have made progress. When the children are fed (and supported), they recover more quickly, showing malnutrition as a key factor in NS". It is noteworthy that since the end of the LRA war and hence closure of the IDP camps in 2009 the people began moving back to their villages and new cases of NS in Northern Uganda began falling dramatically as shown by the graph below showing MoH Survey Report, Sept. 2011.

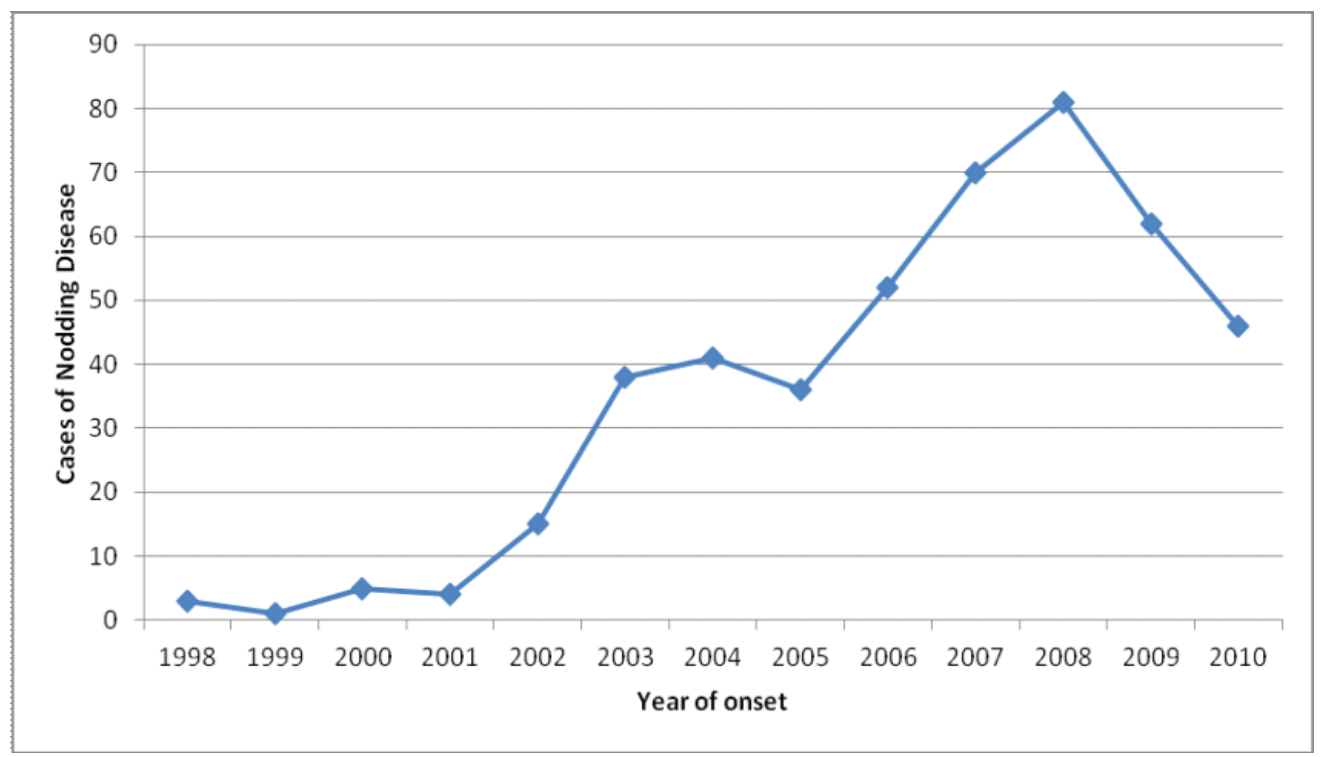

Figure 1: Trend of nodding syndrome in Kitgum district between 1998 and 2010 (Source: MoH Report, Sept. 2011) ${ }^{1}$

\section{Limitations}

This study is a case-series and mainly relied on naturalistic and clinical observations using an eticemic mixed methods approach to report on 6 cases; 3 from the community and 3 representative cases of the 8 cases from Mulago Hospital. No laboratory parameters or measures of body chemistries were relied upon. Thus such socio-anthropological studies could be deemed as subjective and biased as they entail qualitative impressionistic descriptions. The numbers are obviously small and no attempts at statistical analyses were called for. It therefore begs for more robust studies like using case-control, comparative or quasi-experimental approaches to more definitively define the association of exposure 
to war traumata and development of NS. Moreover this study did not explain why the children nod their heads. Lastly, the study was not a clinical trial to answer the question of whether the psychiatric interventions would work for all NS patients. Possible future studies to address these concerns are discussed below.

\section{Conclusion}

Our investigations and observations may suggest that Nodding Syndrome could be a complex syndrome with some children who may have suffered multiple untreated infections and CNS insults resulting in brain damage, epileptic convulsions and mental retardation. However many NS children suffer a form of childhood onset complex post-traumatic stress disorder (PTSD), more appropriately referred to as Developmental Trauma Disorder (DTD) occurring in these chronically war-traumatised children of Northern Uganda, complicated by severe and prolonged depression, psychomotor retardation, fear and anxiety. These children suffered depressive anhedonia and anorexia and also had food shortages, all resulting in malnutrition, wasting and stunted growth with severe avitaminoses. The possible effect of avitaminosis on the genesis of the seizures has not received adequate research attention. Some NS children developed suicidal tendencies and may have died by self drowning, burning or just walking away in the bush and probably killed themselves by any means. Suicide is a common finding in the traumatised peoples of Northern Uganda ${ }^{26}$.

\section{Box 3: Case definition of nodding syndrome ${ }^{3}$}

I. Suspect case: Reported head nodding in a previously normal person. Characterized by repetitive involuntary drops of the head towards the chest on 2 or more occasions.

II. Probable case : Suspect case of head nodding, with:

a.Major criteria: Age of onset of nodding between 3-18 years.Frequency of nodding 5-20/minute. Plus at least one of the following

b. Minor criteria: Other neurological abnormalities (cognitive decline, school dropout due to cognitive / behavioral problems, other seizures or neurological abnormalities). Clustering in space or time with similar cases. Triggering by food, cold weather. Stunting or wasting, Delayed sexual or physical development. Psychiatric symptoms

\section{Confirmed case:}

Probable NS case with:

a. Documented nodding episode

by a trained health care worker or-

Videotaped nodding episode, or-

Video/EEG/EMG 
Table 1: Suggested diagnostic criteria for the psychiatric nodding syndrome

\begin{tabular}{l}
\hline i. Head nodding of at least 2 weeks duration \\
\hline ii. Age of onset of 3-18 years \\
\hline iii. History of repeated exposure to traumatic events/stressors \\
\hline iv. Presence of signs and symptoms of post traumatic stress disorder (PTSD) or developmental trauma \\
disorder(DTD) \\
\hline v.. symptoms are not explainable by any known medical illness and investigations \\
vi.NS Psychiatric type could have any of the following associations:DepressionAnxiety disorderMalnutrition/stunting/ \\
feeding problemsBehavioural/discipline problems Psychotic symptoms Dissociative symptomsCulture/social specific \\
symptoms Psychosomatic/somatoform symptomsPoverty status: social deprivationFailure to progress in \\
schoolCognitive impairment (MR, "IQ) With or without seizures \\
NS Neurological type could have any of the following associations in any combination:Proven ictal activity by \\
EEG and lack of nuchal muscle tone (sternocleidimastoid atonia) as shown by EMG during the head nodding \\
episode. There should be no other explanation for the seizures. A videotaped recording of the head-nodding is \\
complimentary; otherwise a two week reported history of head nodding by a health worker is sufficient.
\end{tabular}

\section{Recommendations}

Prospective cohort studies should be conducted to confirm the suggested NS subtypes and establish their prevalence rates, clinical, psychological and social outcomes as seen in Northern Uganda.

Randomised Clinical Trials are also needed in attempts to finding the definitive treatment for NS based on evidencebased hypotheses including the effects of chronic malnutrition and avitaminoses.

There is also need for brain and psychological autopsy studies of any NS child who dies.

There is a need for ongoing surveillance of NS and the emerging challenges need to be addressed e.g. schooling needs of NS children at their various ages and IQ levels, NS children who get pregnant or drop out of school and need vocational skills or income generating activities. The psychosocial needs and parenting guidance of the caretakers of NS children also need addressing. Lastly the whole Northern Region needs rehabilitative help to catch up with the rest of the country to stop it from becoming marginalized. NS will disappear as the health (including mental health) and wealth of Northern Uganda gets reestablished. Ultimately, Human Rights, peace and conflict resolution are necessary subjects at all levels of our education system to help prevent future war conflicts and their sequelae.

\section{References}

1. Uganda Ministry of Health. A Report On The Burden And Epidemiology of Nodding ddisease in The Districts of Kitgum, Lamwo and Pader in Northern Uganda - August 2010. WHO Sept 1-2, 2011

2. Sejvar JJ, Kakooza AM, Foltz JL, Makumbi I, AtaiOmoruto AD, Malimbo M. Clinical, neurological and electrophysiological features of Nodding Syndrome in Kitgum, Northern Uganda: An observational case series. Lancet Neurology 2013; 12: 166-74.

3. International Scientific Meeting On Nodding syndrome (, July 30 ${ }^{\text {th }}-$ Aug. 02 ${ }^{\text {nd }} 2012$ ): Researchers agree on case definition and establish research agenda. World Health Organization, Regional Office for Africa. http:/ /www.afro.who.int/en/uganda/pressmaterials/item/4826-nodding

4. Korevaar DA Visser BJ (2012): Reviewing the evidence on Nodding syndrome, a mysterious Tropical disorder. International Journal Infectious Diseases http//dx.doi.org/10.1016/ j.ijid.2012.09.015

5. Idro R, Opoka RO, Aanyu HT, Kakooza-Mwesige A, Piloya-Were T, Namusoke H. Nodding syndrome in Ugandan children - Clinical features, Brain imaging and complications; a case series. British Medical Journal Open 2013; 3: e002540.doi; 10.1136/bmjopen-2012

6. Odongkara B, quoted by Wamboka L. Nodding Disease Calls For Collective Action: Commentary in New Vision Newspaper, 2012; 27(099): 10

7. Musisi S, Nakimuli-Mpungu E, Akena DH et al. Psychological Manifestations of Nodding Syndrome in Northern Uganda. African J Traumatic Stress. 2011; 2(1): 56-8.

8. Patel V. Explanatory Models of Mental Illness in sub-Saharan Africa. Social Sciences and Medicine 1995; 40(9): 1291-1298

9. Aall L. Transcultural Research in Mental Health Problems. Review Newsletter. 1962; 13: 54-57 
10. Winkler A. The head nodding syndrome clinical classification And Possible Causes. Epilepsia. 2011; 49(12): 2008-2015.

11. Nyungura LJ, Akim T, Lako (2011): Investigation into Nodding Syndrome in Witto Payam, Western Equatorial State; www.southsudanmedicaljournal.com/archive

12. Lacey M. Nodding Disease: Mystery of Southern Sudan. www.thelancet.com/journals/ laneur/article/PIIS1474-4422(03)00599-4/ fulltext\#

13. Tumwiine J (2012 unpublished): Proceeds of International Scientific Meeting On Nodding Syndrome, Sheraton Hotel, convened by Uganda Ministry of Health, Kampala, July $30^{\text {th }}$ - Aug. 022012

14. Christiana Amanpour (2012): CNN Report on traumatised children, Cable News Network TV program, Oct 2012.

15. Musisi S (2005). War and Mental Health in Africa. In Essentials of Clinical Psychiatry for sub-Saharan Africa. F. Njenga, W. Acuda V. Patel \& M.Maj (Eds) Masson Publications. Milano, Italy

16. Okello J, Onen TS, Musisi S (2005): Psychiatric disorders among War-abducted and Nonabducted Adolescents in Gulu District, Uganda: AComparative Study. African Journal of Psychiatry. 2005; 10:225-231.

17. Musisi S. Mass Trauma and Mental Health in Africa. African Health Sciences Journal. 2004; 4 (2): 3-4

18. Kinyanda E, Musisi S. (2002) War traumatisation and its psychological consequences in women of Gulu district - in Violence against Women and Children: A Review of women's studies, VOL XI Nos 1 and 2. University Center of Women's Studies, University of Philippines Press.

19. Akello G, Richters A, Ovuga E. Children's management of complaints symptomatic of Psychological distress: A Critical analysis of the different approaches in Northern Uganda. African J Traumatic Stress 2010; 1(2): 70-79

20. Bowlby J. Grief and Mourning in infancy and early Childhood. Psychoanalystic study of the Child $1960 ; 15: 9$

21. Bowlby J (1973): Attachment And Loss. Basic Books. NY, NY

22. Harlow HF. Production of depressive Behaviours in young monkeys. J. Autism Child. Schizo. 1971; 1: 246
23. Enoch MD And Trethowan, WH (Eds): Uncommon Psychiatric syndromes, Second Edtition, Year Book Medical Publishers, Chicago, 1979

24. Lishman's Organic Psychiatry: A Textbook of Neuropsychiatry, IVth Edition: David AS, Fleminger S, Kopelman MD, Lovestone S, Mellers JDC - Editors. Wiley-Blackwell Publications. Amazon.co.uk 2009. onlinelibrary.wiley.com/book/10.1002/ 9781444316803

25. Kinyanda E, Nakku J, Oyok OT et al (2009): Suicide in Rural War Affected Northern Uganda. Proceeds Of The XXV World Congress On Suicide Prevention Of The International Association For Suicide Prevention, Montevideo, Uruguay, $27^{\text {th }}-31^{\text {st }}$ October 2009.

26. Ovuga E, Boardman J, Wasserman D. Prevalence of suicide ideation in two districts of Uganda. Archives of Suicide Research 2005; 9(4): 321-332

27. Herman JL. Complex PTSD: A Syndrome in Survivors of Prolonged and Repeated Trauma. Journal of ${ }^{\mathrm{T}}$ raumatic Stress 1992: 5(3):

28. Van Der Kolk BA. Developmental Trauma Disorder. Towards A Rational Diagnosis For Chronically Traumatized Children. Psychiatric Annals 2005; 35: 401-408.

29. Van Der Kolk, BA (2007). The Developmental Impact of Childhood Trauma. In Kirmayer LJ, Lemelson R, and Barad M (Eds.), Understanding Trauma: Integrating Biological, Clinical And Cultural Perspectives (Pp. 224241). New York: Cambridge University Press.

30. Musisi S, Okello ES, Abbo C.Culture And Traditional Healing In Conflict / Post-Conflict Societies. African Journal of Traumatic Stress. 2010; 1(2): 80-85

31. Boehnlein JK.Cultural Interpretations of Physiologic Processes in Post-Traumatic Stress Disorder And Panic Disorder. Transcultural Psychiatry 2001; 38(4):461-467

32. Ovuga EBL, Kipp W, Mungherera M. et al. Epilepsy in a hyper endemic focus of onchocerciasis in rural Western Uganda. East African Medical Journal 1992; 69: 554-556.

33. Piaget J (1954): The Construction of Reality in the Child. Basic Books. NY, NY. USA.

34. Kipp W, Kasolo S, Burnham G. Onchocerciasis and Epilepsy in Uganda. Lancet 1994; 343:183184. 